International Journal of Engineering \& Technology, $7(2.24)(2018) 113-115$
International Journal of Engineering \& Technology
SPC
Website: www.sciencepubco.com/index.php/IJET
Research paper

\title{
Posture Correction for Juvenile Idiopathic Scoliosis Using Flexible Fabric Stretch Sensors
}

\author{
Brightline.A ${ }^{1}$, Malavika Nair ${ }^{2 *}$, Priyanka.B ${ }^{3}$, Prathisha.M ${ }^{4}$, B. Suresh Chandra Kapali ${ }^{5}$ \\ ${ }^{1,2,3}$ UG Students, Department of Biomedical Engineering College, Alpha College of Engineering, Chennai \\ ${ }^{4,5}$ Assistant Professors, Department of Biomedical Engineering College, Alpha College of Engineering, Chennai \\ *Corresponding Author E-mail: maloo1997@gmail.com
}

\begin{abstract}
Our device is being designed to help the juvenile from age 3 to 10 suffering from idiopathic scoliosis, a vertebral deformity that is usually congenital or neuromuscular in nature. The disorder that causes an abnormal lateral curvature of the vertebral column is known as scoliosis. This device will alert the patient by the stimulus given to them whenever the curvature of the bend of spinal cord has reached the threshold value of spinal curve angle on comparing with predetermined value. The sensor used in this device is flexible and thereby can be fixed to the fabrics so that the patients can wear them regularly. By using this device continuously, we can train the patients to maintain good posture for their entire life.
\end{abstract}

Keywords: Idiopathic scoliosis, lateral curvature, spinal cord, posture correction, flex sensor

\section{Introduction}

There are a wide variety of methods which are currently being used for the scoliosis posture correction. This paper deals with the use of flex sensors, microcontroller and a dc motor to help a scoliosis patient to correct his/her posture.

\section{A. Scoliosis}

Human idiopathic scoliosis (IS) is a type of spinal curvature that has no apparent aetiology and occurs during growth in otherwise healthy children. The deformity is characterized by a lateral curvature of the spine, but often occupies all three planes of the human body in the form of sagittal (anterior/posterior) and coronal (left/right) deviation of the spine and axial rotation of the vertebrae (occupying the horizontal plane). Thus, idiopathic scoliosis can be considered a four-dimensional deformity, because progression of the three-dimensional deformity occurs during growth (through time).

\section{B. Problems Faced Due to Scoliosis}

Scoliosis affects the entire skeletal system including the spine, ribs, and pelvis. It impacts upon the brain and central nervous system and affects the body's hormonal and digestive systems. It can deplete the body's nutritional resources and damage its major organs including the heart and lungs.

In severe cases, pressure on your spinal cord may cause loss of coordination in the muscles of your legs, making it difficult for you to walk normally. If your chest is deformed due to the scoliosis, your lungs and heart may be affected, leading to breathing problems, fatigue, and even heart failure.

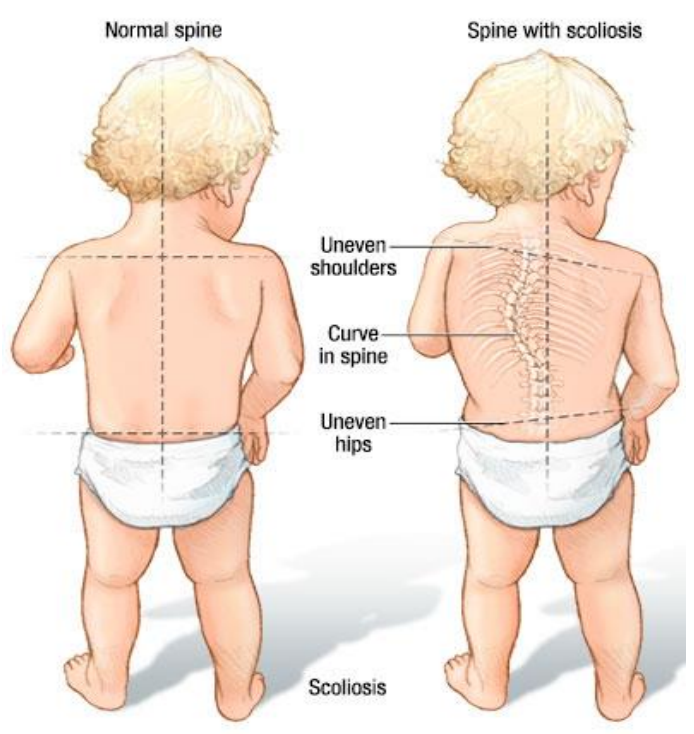

Fig. 1: Deformed spine

\section{Materials and Methodology}

The existing device is used to detect the bending of human's skeleton with the help of images. This is done using a Camera. This makes the system high in cost due to the use of camera. No intimation is provided to the respective user. This fails the person from experiencing damage.

The proposed device overcomes the drawback in the existing device. The human operations are generally carried out by infecting anesthesia to people on Spinal cord. But this might cause various problems for human on the long run. So there are various 
people affected by these type of problems on spinal cords. In order to avoid these type of problems, this device can be used. This device enables monitoring and intimation of the spinal cord of human while they bend to and fro. The system uses capacitive resistive sensors to obtain their bending movement. When such an event is occurred, then a form of alert is provided to the user using vibrations. The vibrations are provided using Motors.

\section{A. Flex Sensors:}

A flex sensor is a sensor that measures the amount of deflection or bending. This flex sensor is a variable resistor. The resistance of the flex sensor increases as the body of the compartment bends. The sensor is stuck to the surface and resistance of sensor element is varied by bending the surface, since the resistance is directly proportional to the amount of the amount of bend.

\section{B. Microcontroller:}

PIC micros have grown to become the most widely used microcontrollers in the 8- bit microcontroller segment.It features 200 ns instruction execution, 256 bytes of EEPROM data memory, self-programming, an ICD, 2 Comparators, 8 channels of 10-bit Analog-to-Digital (A/D) converter, 2 capture/compare/PWM functions, a synchronous serial port that can be configured as either 3-wire SPI or 2-wire I2C bus, a USART, and a Parallel Slave Port.

\section{De Motor:}

A DC motor is designed to run on DC electric power. Two examples of pure DC designs are Michael Faraday's homo polar motor (which is uncommon), and the ball bearing motor, which is (so far) a novelty. By far the most common DC motor types are the brushed and brushless types, which use internal and external commutation respectively to create an oscillating $\mathrm{AC}$ current from the DC source - so they are not purely DC machines in a strict sense. We in our project are using brushed DC Motor, which will operate in the ratings of $12 \mathrm{v}$ DC $0.6 \mathrm{~A}$ which will drive the flywheels in order to make the robot move.

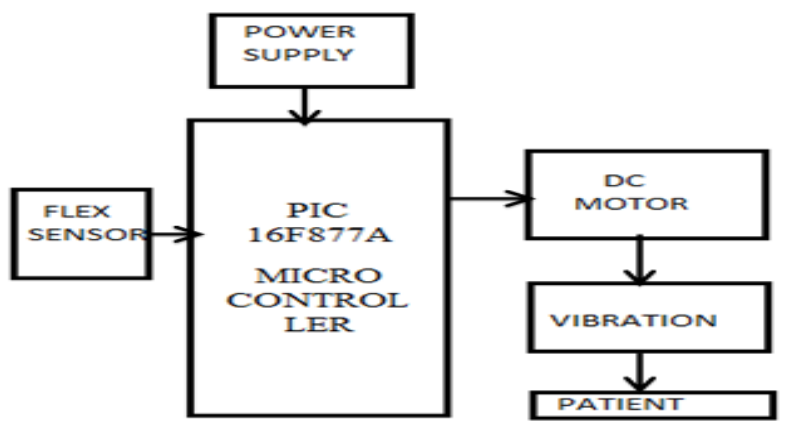

Fig. 2: Block diagram of the posture correction device

\section{Radiologic Evaluation and Classification}

The standing posteroanterior radiographs of the full spine is the standard radiologic evaluation of adolescent idiopathic scoliosis. Follow-up is necessary in those patients with severe curves who are at risk for significant curve progression or require some form of treatment. Any discrepancy in leg length should be corrected with a block placed under the patient's shorter leg when radiographs are taken. One study has shown that long-term management of scoliosis poses no radiograph-related risks to patients, but posteroanterior views assure maximal safety by minimizing radiation to the breasts. The Cobb method is used to measure the degree of scoliosis on the posteroanterior radiograph. In addition to curve degree, physicians should describe curves as "right" or "left," based on their curve convexity. Standard measurement error is 3 to 5 degrees for the same observer and 5 to 7 degrees for different observers when the same end vertebrae are used for measurements. Thus, physicians should use the same end vertebrae for subsequent measurements and assume that some measurement change may be caused by error rather than true curve progression.

\section{E. Measuring Cobb Angle}

To use the Cobb method of measuring the degree of scoliosis, choose the most tilted vertebrae above and below the apex of the curve. The angle between intersecting lines drawn perpendicular to the top of the top vertebrae and the bottom of the bottom vertebrae is the Cobb angle.

The primary goal of treating adolescent idiopathic scoliosis is preventing progression of the curve magnitude. Curves less than 10 to 15 degrees require no active treatment and can be monitored, unless the patient's bones are very immature and progression is likely.

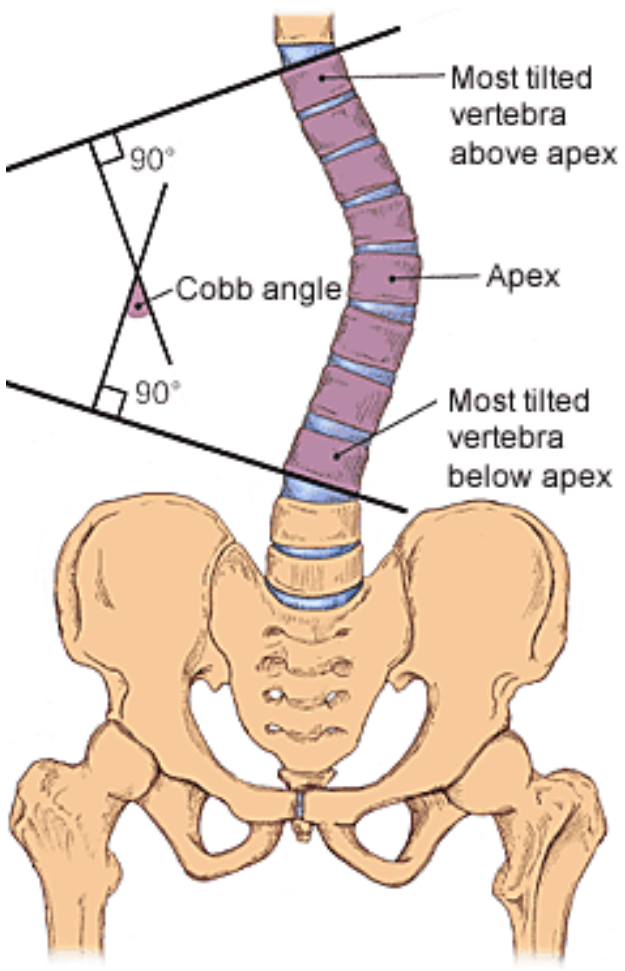

Fig. 3: Cobb angle determination

Moderate curves between 25 and 45 degrees in patients lacking skeletal maturity used to be treated with bracing, but this treatment has never been proven to prevent curve progression. Poor compliance with wearing a brace obviates any potential usefulness of the therapy

\section{F. Sensing}

Flex sensor is used to correct the posture which is flexible and it is fixed with the fabrics such that patient can wear them easily. The threshold degree is set to be 15 degree and thereby when the spine curve is beyond 15 degree flex sensor will sense the posture change and give a mild stimulation to the patient and thereby the patient can return to the normal position.

\section{G. Processing}

The PIC16F877A microcontroller acts as the central part of the unit which will help to process the input data from the sensor and 
provide output signal to the motor if the value obtained deviates beyond the threshold value.

\section{H. Stimulation}

The stimulus or the vibration is given to the patient if proper posture is not maintained. This stimulus will help the patient to understand that they have deviated from the prescribed range and should return back to their normal posture. As the stimulus is a vibration it will not cause any disturbance to the surrounding people and environment.

\section{Predicted Output}

This wearable device will help to correct the posture of the juvenile scoliopathicpatients which will prevent them from acquiring heart diseases and lung disorders.

The angle deviation of the spine is sensed and sent to the PIC controller which will then activate the port which is connected to the motor to enable a vibration to the patient.

\section{Conclusion\& Future Works}

As this posture correction device is wearable and attached to the fabric which is worn by the patient it will provide more comfort and convenience to them when compared to the metallic braces and sensors whch is in contact with the skin.This device will not interrupt any day-to-day activity of the patient and help them to maintain their posture without any external support.

This device can be provided with IOT which can enable the doctors and physicians to monitor the deviations in the angle of the spine whenever required. The whole circuit can be integrated into a small chip which will enable more comfort and efficiency for the patients.

\section{References}

[1] Dr.V.Dooslin Mercy Bai, ArshyaSurendran, "Microcontroller based Scoliosis prevention equipment Using flex sensor", International Innovative Research Journal of Engineering and Technology ISSN NO: 2456-1983,vol 2.

[2] Journal of Neuro Engineering and Rehabilitation Smart portable rehabilitation devices ConstantinosMavroidis,JasonNikitczuketal, Department of Mechanical and Industrial Engineering Northeast University360 Huntington Avenue, Boston MA 02115,USA July 1969.

[3] Effectiveness of audio-biofeedback in posture training for adolescent idiopathic scoliosis patients, WongMS,BrownB.etal,ProsthetOrthotInt.2001APR.3.Angular Biofeedback Device for sitting balance of stroke patients Erbil Dursun, Nigar Hammamei,eatal2005.

[4] Influence of a portable audio-biofeedback device on structure properties of postural sway. Macro Dozza, Becky Chan, etal Department of Electronics, Computer Science, and Systems, University of bologna, Italy and Neurological Science Institute, Oregon Health and Science University, Portland. May 2006.

[5] Behavioral treatment of scoliosis and kyphosis this article is not included in your organization's subscription. However, you may be able to access this article under your organization's agreement with Elsevier. NielsBirbaumer, Neal. Miller, etal, August 2006.

[6] E.Lou,M.J.Moreau,etal ,Capital Health, Glenrose Rehabilitation Hospital, Edmonton, Canada and University of Alberta, Edmonton, Canada.

[7] Scoliosis,IdiopathicPrabhakarRajiah,MD,MBBS,FRCS,Registrar, Department of Radiology, and Manchester Children's University Hospitals ,UK. March 26, 2009.

[8] J.P.Shim,M. Warkentin, J.CourtneyD.Power, R.Shrada, and C.Carlsson, 'past, present, and future of decision support technology', Elsevier, vol.33, pp.111=126, 2009.

[9] B.Kaplan, 'Evaluating Informatics Applications- Clinical Decision Support Systems Literature Review',InternationalJournalofMedicalInformatics, vol.64,no.1,pp.15-37.
[10] C.Lee and M.Wang, 'A Fuzzy Expert Systems for Diabetes Decision Support', IEEE Transaction on Systems, Man, and Cybernetics-Part B: Cybernetics, vol 41,no.1,pp.139-153, 2011.

[11] Archibald D1, KrishnaManohar Vemula2, Anburajan M3, SivaKumar VPR4, Portable biofeedback device for rehabilitating children with scoliosis between ages of 3-10 years and their posture correction,ICECTECH.2011.5941744 .

[12] Barry Dworkin, Neal E. Millert, SUSAN Dworkin, NielsBirbaumert, Michael L. Brines, Saran Jonas, Edwards P. Schwentker, And Jacob J. Grahami, Behavioral method for the treatment of idiopathic scoliosis, Vol. 82, pp. 2493-2497, April 1985 MedicalSciences

[13] Kristen Fay Gorman,The curvebackguppy as a model for human idiopathic-type spinal curvature.

[14] Edmond Lou, Ph.D., Michael Bazzarelli,B.Sc, Doug Hill, MBA, Nelson Dude, Ph.D. ,A low power accelerometer used to improve posture

[15] Mavroidis,JasonNikitczuketal, Department of Mechanical and Industrial Engineering Northeast University360 Huntington Avenue, Boston.Journal of Neuro Engineering and Rehabilitation Smart portable rehabilitation devices Constantinos MA 02115,USA July 1969.

[16] Angular Biofeedback Device for sitting balance of stroke patients Erbil Dursun, NigarHammamei, etal2005.

[17] Influence of a portable audio-biofeedback device on structure properties of postural sway. Macro Dozza, Becky Chan, etal Department of Electronics, Computer Science, and Systems, University of bologna, Italy and Neurological Science Institute, Oregon Health and Science University, Portland. May 2006. 